\title{
Archives of Ecotoxicology
}

\author{
Journal homepage: https://office.scicell.org/index.php/AE
}

\section{In Vitro Antimicrobial Screening of Momordica charantia extracts against Multidrug-Resistant Bacterial Strains}

\author{
Hina Qamara, Sumbul Rehmanb, Saimul Deharc, Touseef Hussain ${ }^{d^{*}}$ \\ a Department of Zoology, Chaudhary Charan Singh University, Meerut, Uttar Pradesh (250001) India \\ ${ }^{b}$ Department of Ilmul Advia (Unani Pharmacology), A.K. Tibbiya College, Aligarh Muslim University, Aligarh, Uttar Pradesh (202002) India \\ ${ }^{c}$ Rajputana Unani Medical College Hospital \& Research Centre, Jaipur, Rajasthan (302017) India \\ ${ }^{d}$ Deptartment of Botany, Aligarh Muslim University, Aligarh, Uttar Pradesh (202002) India
}

\section{Article info}

Received 6 February 2020

Revised 27 February 2020

Accepted 27 May 2020

Published online 3 June 2020

Regular article

\section{Keywords:}

Multidrug-resistance

Ethyl acetate fraction

Bacteria

E.coli

S.aureus

M. charantia

Successive extraction

Abstract

A comparative study for screening the antibiotic potential of Momordica charantia successive extracts in vitro against eleven multidrug-resistant bacterial strains. Momordica charantia fresh fruit was extracted successively in different solvents in the order of increasing polarity from hexane to aqueous followed by screening against eleven antibiotic-resistant bacterial strains including both Gram-positive and Gramnegative bacterial strains using Kirby-Bauer's disk diffusion and agar well diffusion method. A study on Staphylococcus epidermidis and Corynebacterium xerosis are reported for the first time. Significant inhibitory activity was noted against most of the resistant human pathogenic strains. Findings reported that ethyl acetate fraction shows the highest zone of inhibition while hexane, petroleum ether, chloroform and aqueous extract were almost resistant. The antibacterial efficacy of Momordica charantia is found to be significant. It's also concluded that the controversy on the antibacterial activity of Momordica charantia fruit extract in different solvents is based on several factors like the solvent used for extraction, plant part, concentration, method of extraction, etc. M. charantia extracts could be used as an alternative anti-microbial to replace antibiotics for treating a broad spectrum of multidrug-resistant bacterial diseases.
\end{abstract}

\section{Introduction}

In the $21^{\text {st }}$ century, antimicrobial resistance is the greatest challenge to public health and threatens modern medicine where common infections could become more deadly. Antimicrobial resistance is the ability of a microbe to resist the effects of medication that once could successfully treat the microbe. The discovery of antibiotics was one of the most important developments in medicine but misuse and overuse of antibiotics to treat viral infections, use of broad-spectrum antibiotics and as growth promoters in animals leads to emergence of bacteria that have evolved resistance to multiple antibiotics and gave birth to superbugs and multidrug-resistant microbes in the environment (Sharma V.K, 2016). The persistence of these antibiotic-resistant microbes not only creates increased opportunities to transfer the resistance genes to associated susceptible bacteria but also eventually lead to entry into the human food chain (Founou, et al., 2016).

Globally, new resistance mechanisms are continuously being emerging and spreading. This has triggered initiatives worldwide to develop novel and more effective strategies to counteract antimicrobial resistance. Today in the synthetic world where common infection could become a threat, returning towards herbal therapy is a safe and noninvasive method for the betterment of healthcare. Since ages, natural products that include medicinal plants have been used to prevent, cure and treat multiple diseases. Herbal products from medicinal plants provide unlimited opportunities for new drug leads either as pure compounds or as standardized extracts. They possess a huge diversity of bioactive compounds having safer and more efficient therapeutic potential. As natural compounds have relatively better safety profile they are gaining a lot of attention in drug discovery programs. World Health Organization (WHO) reported about $80 \%$ of the world's population that utilizes traditional medicine as their first line of therapy (WHO, 20022005). But today people are more focused on modern medicine. Though both therapies have several pros and cons. So, to find solutions to the severest problem facing the health system by intelligently selecting, harmonization of traditional and modern medicine promote the best care for patients.

Momordica charantia commonly called bitter gourd belongs to Cucurbitaceae family. It is widely distributed in tropical and subtropical regions of the world. M. charantia is frequently used in the traditional medicine due to its anti-bacterial, anti-viral, anti-tumor, anti-oxidant, anti-diabetes, antilipolytic, antiinflammatory, anthelmintic, immunomodulatory and hepatoprotective properties (Shuo Jia, et al., 2017). Although hundreds of plant species have been evaluated for phytochemical profile and screened for antimicrobial properties, the vast majority of the plants have not been 
adequately screened and evaluated. In the present study successive extraction of Momordica charantia was evaluated and each fraction was screened against several multidrug-resistant bacterial strains along with some novel findings.

\section{Material and methods}

All solvents used were of HPLC grade obtained from SigmaAldrich. Nutrient agar, Nutrient broth medium, and antimicrobial disks were purchased from Hi-media Labs, Mumbai. The bacterial cultures were kindly provided by Microbiology Lab, Department of Ilmul Advia and Jawaharlal Nehru Medical College and Hospital, Aligarh Muslim University, Aligarh.

\subsection{Plant Material}

Fresh Momordica charantia (MC) fruit was procured from the market, authenticated with the botanical literature available and identification made accordingly by pharmacognosy expert. The whole MC fruit comprising pericarp, pulp and seeds was washed thoroughly and subjected to grinding in a mixer to get a homogenized mixer.

\subsection{Successive Extraction and Isolation of Chemical Constituents}

The homogenized mixer of fresh MC fruit was subjected to successive extraction sequentially from hexane, petroleum ether, ethyl acetate, chloroform, acetone, n-butanol, ethanol and methanol to water (each 1:3 w/v ratio). Hexane and petroleum fractions were subjected to 24 hours maceration with continuous stirring while rest fractions were subjected to soxhlet hot extraction. Each fraction collected separately after filtration followed by drying.

\subsection{Alcoholic and Aqueous Extraction}

For alcoholic extraction the fresh MC fruit paste $(250 \mathrm{~g})$ was subjected to extraction by heating to reflux with $95 \%$ ethanol (plant: solvent $1: 2, \mathrm{~m} / \mathrm{v}$ ) in a soxhlet apparatus at $50^{\circ} \mathrm{C}$ for $5 \mathrm{hrs}$. Then filtered the ethanolic extract (HEE), collected the filtrate and reduce the concentration to a small volume by drying. Similarly, aqueous extraction (HAE) was done separately.

\subsection{Antimicrobial Assay}

Antibiotic susceptibility testing for multidrug-resistance: Multidrug-resistant testing for all Gram-positive and Gramnegative clinical strains was determined using Kirby-Bauer disk diffusion method according to the Clinical and Laboratory Standards Institute (CLSI 2006) guidelines against certain antibiotics, namely, Cefixime $(10 \mu \mathrm{g})$, Amoxyclav $(10 \mu \mathrm{g})$, Cefotaxime $(10 \mu \mathrm{g})$ and Methicillin $(10 \mu \mathrm{g})$, and plates were incubated at $37^{\circ} \mathrm{C}$ for 24 hours. The next day, the diameters of the zone of inhibition around the discs were measured.

Drug susceptibility test: The susceptibility of different extracts of $M$. charantia against multidrug-resistant bacterial strains was determined using Kirby-Bauer's disk diffusion and agar well diffusion method according to CLSI (Clinical Laboratory Standard Institute) Guidelines as mentioned above. Seven Grampositive (Staphylococcus aureus, Streptococcus mutans, Streptococcus pyogenes, Streptococcus viridans, Staphylococcus epidermidis, Corynebacterium xerosis, and Bacillus cereus) and four Gram-negative (Escherichia coli, Klebsiella pneumonia, Pseudomonas aeruginosa, and Proteus vulgaris) multidrugresistant bacterial strains were used. About $50 \mu \mathrm{l}$ of the test sample was used against each strain swabbed on nutrient agar plates followed by incubation at $37^{\circ} \mathrm{C}$ for $24 \mathrm{hrs}$. Different extracting solvents were used as negative control while for positive control $10 \mu \mathrm{g}$ Streptomycin disk for Gram-positive and $10 \mu \mathrm{g}$ Norfloxacin disk for Gram-negative strains were used. Antimicrobial activity was assessed using the zone of inhibition (ZoI) measured after the incubation period against each tested micro-organisms and was compared with the standard used and analyzed.

\subsection{Statistical Analysis}

The experiment was performed in a triplet, compared with the standard used and analyzed statistically using graph-paid software by Tukey Kramer Comparison test, One way ANOVA.

\section{Results}

The findings show that all Gram-positive and Gram-negative clinical strains are resistant against antibiotics, namely, Cefixime $(10 \mu \mathrm{g})$, Amoxyclav $(10 \mu \mathrm{g})$, Cefotaxime $(10 \mu \mathrm{g})$ and Methicillin $(10 \mu \mathrm{g})$. The sequential successive extraction in ascending order of polarity from hexane to aqueous extract showed different zone of inhibitions against each strain (Fig.1) (Table.1). Hexane, petroleum ether, chloroform and aqueous extract are almost resistant to all eleven bacterial strains while ethyl acetate, acetone, n-butanol, the ethanolic and methanolic extract showed significant activity against all bacterial strains. On average, it's observed that as the polarity of the solvent increasing from ethyl acetate to methanol the antimicrobial activity of the extract decreases subsequently. Ethyl acetate crude extracts exhibited a considerably broader antimicrobial activity compared to other extracts. The maximum ZoI of $30 \mathrm{~mm}$ was produced by ethyl acetate fraction against Streptococcus pyogenes. Besides, the ethanolic extract of successive extraction showed lesser antibacterial activity than extract obtained following nonsuccessive extraction. Further, to the best of my knowledge antibacterial activity of $M$. charantia against Staphylococcus epidermidis and Corynebacterium xerosis is reported for the first time.

\section{Discussion}

Cefixime and cefotaxime are third generations broad-spectrum antibiotics while methicillin is narrow-spectrum $\beta$-lactam antibiotic. Amoxyclav is a combination of a salt of amoxicillin and clavulanic acid. Amoxicillin works as an antibiotic and interferes with the bactericidal effect by disrupting the cell wall of bacteria while clavulanic acid reduces resistance and acts as a $\beta$ lactamase inhibitor. Numerous extended-spectrum $\beta$ lactamases (ESBLs) cause resistance to the above antibiotics and other newer cephalosporin (Bush, K, et al., 1995). Findings reported resistance to all the above antibiotics. This may be due to the high prevalence of extended-spectrum $\beta$-lactamases and metallo- $\beta$-lactamases amongst the bacteria. The possible reason for acquiring $\beta$-lactam resistance in bacteria includes the production of high $\beta$-lactamase, impermeability of outer membrane and active efflux mediated by RND-type efflux systems (Poole K, 2011).

To date, there is a controversy on the antibacterial activity of Momordica charantia fruit. Several studies reported antibacterial activity while others not. Earlier antibacterial activity of different plant parts has been reported against several Gram-positive and Gram-negative bacteria that are under the results gained (M. Asan Ozusaglam et al., 2013; P. Supraja et al., 2013; Yang Lin Yeo, et al., 2014 and Gulsum Yaldiza, et al., 2015). However, some of the researchers reported antibacterial activity of hexane, petroleum ether and aqueous extract (YinYin Chia et al., 2011; Abid Mahmood 2012; Yang Lin Yeo, et al., 2014) but in our study the results were negative. 
Negative results were supported by the previous findings in which petroleum ether crude extracts of fruits showed no inhibitory activity against Escherichia coli and Pseudomonas aeruginosa (K. D. Mwambete 2009). The bitter melon extract was obtained from its interior, middle, and outside skin and mixed with sterile distilled water, the extract showed no inhibition against $S$. aureus and E. coli (Debolina Ghosh, 2014). Antibacterial activity of fresh juice of skin and pulp of $M$. charantia against different bacterial strains was reported earlier (Sabahat Saeed and Perween Tariq, 2005) with ZoI between $14-17 \mathrm{~mm}$. Beside, deseeded fresh fruit water and methanolic extract showed no inhibitory activity against Methicillinresistant Staphylococcus aureus and Pseudomonas aeruginosa while showed antibacterial activity against Escherichia coli and Salmonella enteric (Yeh-Lin Lu et al., 2011). Also, several leave extracts of the plant were reported too. Aqueous, methanolic and ethanolic extracts of Momordica charantia leaves showed antibacterial activity (G. Leelaprakash et al., 2011; S. B. Mada et al., 2013; Adegbola, et al., 2016). In the present study while going in deep and co-relating our results with the previous studies it's come to an end that the antibacterial activity of Momordica charantia is based on several factors.

Hexane and petroleum ether both have a polarity index of nearzero $(\mathrm{P}=0.1)$. The extract of $M$. charantia in both solvents shows resistance, as might be antimicrobial compounds that are responsible for inhibitions are not extracted. However previous findings showed that $E$. coli was susceptible to hexane extract (Yang Lin Yeo, et al., 2014). It might be possible that the antimicrobial compounds present in the hexane and petroleum ether with lower polarity index tend to be extracted at longer maceration time or by using a hot extraction method. Similarly, though the polarity index of chloroform is high $\left(\mathrm{P}^{\prime}=4.1\right)$ but extraction time and method limit its activity. Ethyl-acetate with polarity index 4.4 is widely used as an extraction solvent. Extraction in ethyl acetate showed the highest antimicrobial activity against all bacterial strains with $\mathrm{ZoI}$ ranging from 20-30 $\mathrm{mm}$. This indicates that the active ingredients of the plant are more readily dissolved and extracted in ethyl-acetate compared with other solvents used. Next, to ethyl acetate, acetone with polarity index 5.1 showed significant activity. Though the polarity index of acetone is higher than ethyl acetate during successive extraction most of the phytochemicals are already extracted in ethyl acetate. Preceding, solvents such as $\mathrm{n}$ - butanol $(\mathrm{P}=3.9)$, ethanol $\left(\mathrm{P}^{\prime}=4.3\right)$, methanol $\left(\mathrm{P}^{\prime}=5.1\right)$ and distilled water $(\mathrm{P}=10.2)$ extracted lower phytochemical profiles and thus show no or very little inhibition against different bacterial strains. Findings showed that intermediate-polar solvent systems used in extracting antimicrobial compounds from fruit of Momordica charantia L showed significant activity.

Further, when a comparative study on the ethanolic and aqueous extracts following successive extraction and non-successive extraction protocol was done, it's found that aqueous extracts of both the procedure do not show any antibacterial activity while the ethanolic extract of the non-successive extract showed more activity than successive extract. This might be because during successive extraction phytochemicals such as flavonols and phenols are extracted by solvents such as ethyl acetate and acetone so, the ethanolic extract shows less activity (Table. 2).
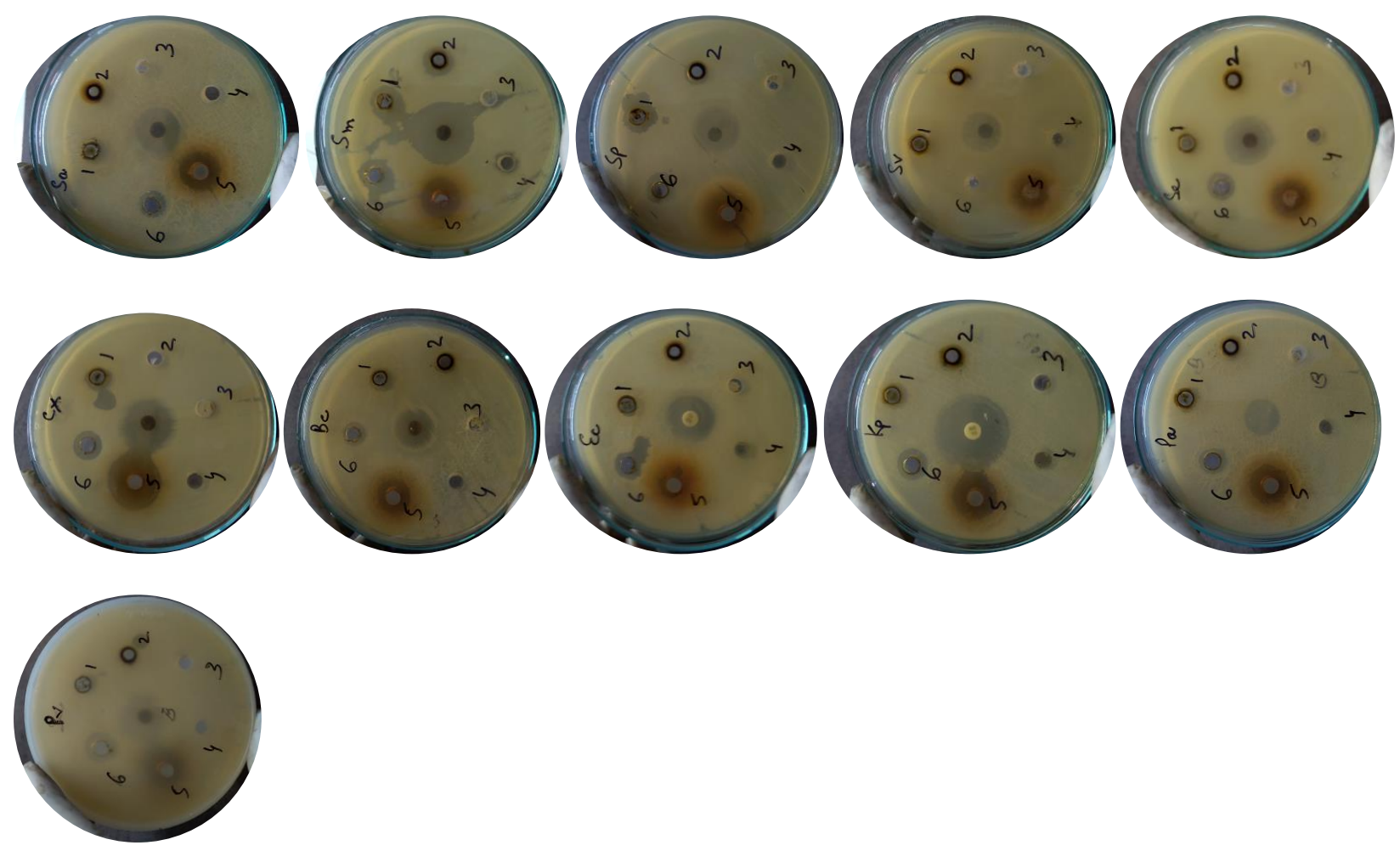

Figure 1. In vitro antibacterial activity of $M$. charantia extracts 
Table 1. Drug susceptibility readings against various multidrug-resistant microbial strains

\begin{tabular}{|c|c|c|c|c|c|c|c|c|c|c|}
\hline \multirow{2}{*}{\multicolumn{2}{|c|}{$\begin{array}{c}\text { Name of Microbial } \\
\text { Strains }\end{array}$}} & \multirow{2}{*}{\multicolumn{9}{|c|}{$\begin{array}{c}\text { Zone of Inhibition (in mm) } \\
\text { [ Mean } \pm \text { SEM (SD)] }\end{array}$}} \\
\hline & & & & & & & & & & \\
\hline & & HE & $\mathbf{P E}$ & EAE & $\mathbf{C E}$ & ACE & BE & $\mathbf{E E}$ & ME & AE \\
\hline \multirow{7}{*}{ 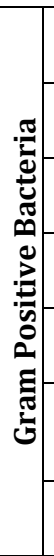 } & $\begin{array}{l}\text { Staphylococcus } \\
\text { aureus }\end{array}$ & $\begin{array}{c}6.6 \pm 0.3 \\
(0.57)\end{array}$ & $\begin{array}{c}6.3 \pm 0.3 \\
(0.57)\end{array}$ & $\begin{array}{c}27.6 \pm 0.3 \\
(0.57)\end{array}$ & $\begin{array}{c}6.6 \pm 0.3 \\
(0.57)\end{array}$ & $\begin{array}{c}19.6 \pm 0.3 \\
(0.57)\end{array}$ & $\begin{array}{c}10.6 \pm 0.3 \\
(0.57)\end{array}$ & $\begin{array}{c}6.6 \pm 0.3 \\
(0.57) \\
\end{array}$ & $\begin{array}{c}6.6 \pm 0.3 \\
(0.57) \\
\end{array}$ & $\begin{array}{c}6.3 \pm 0.3 \\
(0.57)\end{array}$ \\
\hline & $\begin{array}{l}\text { Streptococcus } \\
\text { mutans }\end{array}$ & $\begin{array}{c}6.3 \pm 0.3 \\
(0.57) \\
\end{array}$ & $\begin{array}{c}6.6 \pm 0.3 \\
(0.57) \\
\end{array}$ & $\begin{array}{c}28.6 \pm 0.3 \\
(0.57)\end{array}$ & $\begin{array}{c}6.6 \pm 0.3 \\
(0.57) \\
\end{array}$ & $\begin{array}{c}14.6 \pm 0.3 \\
(0.57)\end{array}$ & $\begin{array}{c}10.6 \pm 0.3 \\
(0.57)\end{array}$ & $\begin{array}{c}11.6 \pm 0.3 \\
(0.57)\end{array}$ & $\begin{array}{c}11.6 \pm 0.3 \\
(0.57)\end{array}$ & $\begin{array}{l}6.6 \pm 0.3 \\
(0.57)\end{array}$ \\
\hline & $\begin{array}{l}\text { Streptococcus } \\
\text { pyogenes }\end{array}$ & $\begin{array}{c}6.3 \pm 0.3 \\
(0.57)\end{array}$ & $\begin{array}{c}6.3 \pm 0.3 \\
(0.57)\end{array}$ & $\begin{array}{c}30.6 \pm 0.3 \\
(0.57)\end{array}$ & $\begin{array}{c}6.6 \pm 0.3 \\
(0.57)\end{array}$ & $\begin{array}{c}22.6 \pm 0.3 \\
(0.57)\end{array}$ & $\begin{array}{c}10.6 \pm 0.3 \\
(0.57)\end{array}$ & $\begin{array}{c}11.6 \pm 0.3 \\
(0.57)\end{array}$ & $\begin{array}{c}10.6 \pm 0.3 \\
(0.57)\end{array}$ & $\begin{array}{c}6.6 \pm 0.3 \\
(0.57)\end{array}$ \\
\hline & $\begin{array}{l}\text { Streptococcus } \\
\quad \text { viridans }\end{array}$ & $\begin{array}{c}6.6 \pm 0.3 \\
(0.57)\end{array}$ & $\begin{array}{c}6.3 \pm 0.3 \\
(0.57)\end{array}$ & $\begin{array}{c}26.6 \pm 0.3 \\
(0.57)\end{array}$ & $\begin{array}{c}6.6 \pm 0.3 \\
(0.57)\end{array}$ & $\begin{array}{c}21.6 \pm 0.3 \\
(0.57)\end{array}$ & $\begin{array}{c}11.6 \pm 0.3 \\
(0.57)\end{array}$ & $\begin{array}{c}7.6 \pm 0.3 \\
(0.57)\end{array}$ & $\begin{array}{c}6.6 \pm 0.3 \\
(0.57)\end{array}$ & $\begin{array}{c}6.6 \pm 0.3 \\
(0.57)\end{array}$ \\
\hline & $\begin{array}{l}\text { Staphylococcus } \\
\text { epidermidis }\end{array}$ & $\begin{array}{c}6.6 \pm 0.3 \\
(0.57)\end{array}$ & $\begin{array}{c}6.6 \pm 0.3 \\
(0.57)\end{array}$ & $\begin{array}{c}25.6 \pm 0.3 \\
(0.57)\end{array}$ & $\begin{array}{c}6.6 \pm 0.3 \\
(0.57)\end{array}$ & $\begin{array}{c}15.6 \pm 0.3 \\
(0.57)\end{array}$ & $\begin{array}{c}12.6 \pm 0.3 \\
(0.57)\end{array}$ & $\begin{array}{c}12.6 \pm 0.3 \\
(0.57) \\
\end{array}$ & $\begin{array}{c}12.6 \pm 0.3 \\
(0.57)\end{array}$ & $\begin{array}{c}6.6 \pm 0.3 \\
(0.57)\end{array}$ \\
\hline & $\begin{array}{c}\text { Corynebacterium } \\
\text { xerosis }\end{array}$ & $\begin{array}{c}6.3 \pm 0.3 \\
(0.57)\end{array}$ & $\begin{array}{c}6.6 \pm 0.3 \\
(0.57)\end{array}$ & $\begin{array}{c}27.6 \pm 0.3 \\
(0.57)\end{array}$ & $\begin{array}{c}6.6 \pm 0.3 \\
(0.57)\end{array}$ & $\begin{array}{c}16.6 \pm 0.3 \\
(0.57)\end{array}$ & $\begin{array}{c}6.6 \pm 0.3 \\
(0.57)\end{array}$ & $\begin{array}{c}8.6 \pm 0.3 \\
(0.57)\end{array}$ & $\begin{array}{c}8.3 \pm 1.3 \\
(2.3)\end{array}$ & $\begin{array}{c}6.6 \pm 0.3 \\
(0.57)\end{array}$ \\
\hline & Bacillus cereus & $\begin{array}{c}6.6 \pm 0.3 \\
(0.57)\end{array}$ & $\begin{array}{c}6.6 \pm 0.3 \\
(0.57)\end{array}$ & $\begin{array}{c}27.6 \pm 0.3 \\
(0.57)\end{array}$ & $\begin{array}{c}6.6 \pm 0.3 \\
(0.57)\end{array}$ & $\begin{array}{c}14.6 \pm 0.3 \\
(0.57)\end{array}$ & $\begin{array}{c}11.6 \pm 0.3 \\
(0.57)\end{array}$ & $\begin{array}{c}8.6 \pm 0.3 \\
(0.57)\end{array}$ & $\begin{array}{l}8 \pm 1.0 \\
(1.7)\end{array}$ & $\begin{array}{c}6.6 \pm 0.3 \\
(0.57)\end{array}$ \\
\hline \multirow{4}{*}{$\begin{array}{l}0 \\
. \\
0 \\
0 \\
\mathbf{0} \\
\mathbf{0} \\
\mathbf{5} \\
\mathbf{5}\end{array}$} & Escherichia coli & $\begin{array}{c}6.3 \pm 0.3 \\
(0.57)\end{array}$ & $\begin{array}{c}6.3 \pm 0.3 \\
(0.57)\end{array}$ & $\begin{array}{c}20.6 \pm 0.3 \\
(0.57)\end{array}$ & $\begin{array}{c}6.3 \pm 0.3 \\
(0.57)\end{array}$ & $\begin{array}{c}13.6 \pm 0.3 \\
(0.57)\end{array}$ & $\begin{array}{c}11.6 \pm 0.3 \\
(0.57)\end{array}$ & $\begin{array}{c}7.6 \pm 0.3 \\
(0.57) \\
\end{array}$ & $\begin{array}{c}8.3 \pm 1.3 \\
(2.3)\end{array}$ & $\begin{array}{c}6.3 \pm 0.3 \\
(0.57)\end{array}$ \\
\hline & $\begin{array}{c}\text { Klebsiella } \\
\text { pneumonia }\end{array}$ & $\begin{array}{c}6.3 \pm 0.3 \\
(0.57)\end{array}$ & $\begin{array}{c}6.6 \pm 0.3 \\
(0.57)\end{array}$ & $\begin{array}{c}27.6 \pm 0.3 \\
(0.57)\end{array}$ & $\begin{array}{c}7.3 \pm 0.3 \\
(0.57)\end{array}$ & $\begin{array}{c}15 \pm 1.0 \\
(1.7)\end{array}$ & $\begin{array}{c}11.6 \pm 0.3 \\
(0.57)\end{array}$ & $\begin{array}{c}10.6 \pm 0.3 \\
(0.57)\end{array}$ & $\begin{array}{c}11.3 \pm 0.8 \\
(1.5)\end{array}$ & $\begin{array}{l}6.3 \pm 0.3 \\
3(0.57)\end{array}$ \\
\hline & $\begin{array}{l}\text { Pseudomonas } \\
\text { aeruginosa }\end{array}$ & $\begin{array}{c}6.6 \pm 0.3 \\
(0.57)\end{array}$ & $\begin{array}{l}6.3 \pm 0.3 \\
(0.57)\end{array}$ & $\begin{array}{c}26.6 \pm 0.3 \\
(0.57)\end{array}$ & $\begin{array}{c}6.3 \pm 0.3 \\
(0.57)\end{array}$ & $\begin{array}{c}17.6 \pm 0.3 \\
(0.57)\end{array}$ & $\begin{array}{c}10.6 \pm 0.3 \\
(0.57)\end{array}$ & $\begin{array}{c}7.6 \pm 0.3 \\
(0.57) \\
\end{array}$ & $\begin{array}{c}11.6 \pm 0.3 \\
(0.57)\end{array}$ & $\begin{array}{c}6.3 \pm 0.3 \\
(0.57)\end{array}$ \\
\hline & Proteus vulgaris & $\begin{array}{c}6.6 \pm 0.3 \\
(0.57)\end{array}$ & $\begin{array}{c}6.3 \pm 0.3 \\
(0.57)\end{array}$ & $\begin{array}{c}24.6 \pm 0.3 \\
(0.57)\end{array}$ & $\begin{array}{c}7.3 \pm 0.3 \\
(0.57)\end{array}$ & $\begin{array}{c}17.6 \pm 0.3 \\
(0.57)\end{array}$ & $\begin{array}{c}11.6 \pm 0.3 \\
(0.57)\end{array}$ & $\begin{array}{c}10.6 \pm 0.3 \\
(0.57)\end{array}$ & $\begin{array}{c}13.6 \pm 0.3 \\
(0.57)\end{array}$ & $\begin{array}{c}6.6 \pm 0.3 \\
(0.57)\end{array}$ \\
\hline
\end{tabular}

Legend: Successive extraction= HE: Hexane extract, PE: Petroleum ether extract, EAE: Ethyl acetate extract, CE: Chloroform extract, AcE: Acetone extract, BE: n-butanol extract, EE: Ethanol extract, ME: Methanol extract, AE: Aqueous extract

Table 2. A comparative study on successive and non-successive ethanolic and aqueous extracts against various multidrug-resistant microbial strains.

\begin{tabular}{|c|c|c|c|c|c|c|c|}
\hline & \multirow{2}{*}{$\begin{array}{c}\text { Name of Microbial } \\
\text { Strains }\end{array}$} & \multicolumn{6}{|c|}{$\begin{array}{c}\text { Zone of Inhibition (in mm) } \\
\text { [Mean } \pm \text { SEM (SD)] }\end{array}$} \\
\hline & & $\begin{array}{l}\text { Ethanolic } \\
\text { (SE) }\end{array}$ & $\begin{array}{l}\text { Ethanolic } \\
\text { (NSE) }\end{array}$ & $\begin{array}{l}\text { Aqueous } \\
\text { (SE) }\end{array}$ & $\begin{array}{l}\text { Aqueous } \\
\text { (NSE) }\end{array}$ & $\begin{array}{l}\text { Positive } \\
\text { control }\end{array}$ & $\begin{array}{l}\text { Negative } \\
\text { control }\end{array}$ \\
\hline \multirow{7}{*}{ 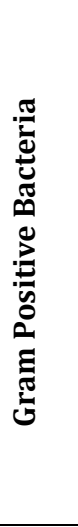 } & Staphylococcus aureus & $\begin{array}{l}6.6 \pm 0.3 \\
(0.57)\end{array}$ & $\begin{array}{l}10.3 \pm 0.8 \\
(0.57)\end{array}$ & $\begin{array}{l}6.3 \pm 0.3 \\
(0.57)\end{array}$ & $\begin{array}{l}6.6 \pm 0.3 \\
(0.57)\end{array}$ & $\begin{array}{l}18.3 \pm 0.3 \\
(0.57)\end{array}$ & $\begin{array}{l}6.6 \pm 0.3 \\
(0.57)\end{array}$ \\
\hline & Streptococcus mutans & $\begin{array}{l}11.6 \pm 0.3 \\
(0.57)\end{array}$ & $\begin{array}{l}12.3 \pm 0.3 \\
(0.57)\end{array}$ & $\begin{array}{l}6.6 \pm 0.3 \\
(0.57)\end{array}$ & $\begin{array}{l}6.6 \pm 0.3 \\
(0.57)\end{array}$ & $\begin{array}{l}27.3 \pm 0.3 \\
(0.57)\end{array}$ & $\begin{array}{l}6.3 \pm 0.3 \\
(0.57)\end{array}$ \\
\hline & Streptococcus pyogenes & $\begin{array}{l}11.6 \pm 0.3 \\
(0.57)\end{array}$ & $\begin{array}{l}19.3 \pm 0.3 \\
(0.57)\end{array}$ & $\begin{array}{l}6.6 \pm 0.3 \\
(0.57)\end{array}$ & $\begin{array}{l}6.6 \pm 0.3 \\
(0.57)\end{array}$ & $\begin{array}{l}19.3 \pm 0.8 \\
(0.57)\end{array}$ & $\begin{array}{l}6.3 \pm 0.3 \\
(0.57)\end{array}$ \\
\hline & Streptococcus viridans & $\begin{array}{l}7.6 \pm 0.3 \\
(0.57) \\
\end{array}$ & $\begin{array}{l}19.3 \pm 0.8 \\
(0.57)\end{array}$ & $\begin{array}{l}6.6 \pm 0.3 \\
(0.57)\end{array}$ & $\begin{array}{l}6.3 \pm 0.3 \\
(0.57)\end{array}$ & $\begin{array}{l}12.3 \pm 0.3 \\
(0.57)\end{array}$ & $\begin{array}{l}6.3 \pm 0.3 \\
(0.57)\end{array}$ \\
\hline & $\begin{array}{l}\text { Staphylococcus } \\
\text { epidermidis }\end{array}$ & $\begin{array}{l}12.6 \pm 0.3 \\
(0.57)\end{array}$ & $\begin{array}{l}14.3 \pm 0.6 \\
(0.57)\end{array}$ & $\begin{array}{l}6.6 \pm 0.3 \\
(0.57) \\
\end{array}$ & $\begin{array}{l}6.3 \pm 0.3 \\
(0.57) \\
\end{array}$ & $\begin{array}{l}12.3 \pm 0.3 \\
(0.57)\end{array}$ & $\begin{array}{l}6.3 \pm 0.3 \\
(0.57) \\
\end{array}$ \\
\hline & $\begin{array}{l}\text { Corynebacterium } \\
\text { xerosis }\end{array}$ & $\begin{array}{l}8.6 \pm 0.3 \\
(0.57) \\
\end{array}$ & $\begin{array}{l}19.3 \pm 0.6 \\
(0.57)\end{array}$ & $\begin{array}{l}6.6 \pm 0.3 \\
(0.57) \\
\end{array}$ & $\begin{array}{l}6.3 \pm 0.3 \\
(0.57)\end{array}$ & $\begin{array}{l}15.3 \pm 0.6 \\
(0.57)\end{array}$ & $\begin{array}{l}6.3 \pm 0.3 \\
(0.57) \\
\end{array}$ \\
\hline & Bacillus cereus & $\begin{array}{l}8.6 \pm 0.3 \\
(0.57) \\
\end{array}$ & $\begin{array}{l}12.3 \pm 0.6 \\
(0.57)\end{array}$ & $\begin{array}{l}6.6 \pm 0.3 \\
(0.57) \\
\end{array}$ & $\begin{array}{l}6.6 \pm 0.3 \\
(0.57)\end{array}$ & $\begin{array}{l}13.3 \pm 0.6 \\
(1.15)\end{array}$ & $\begin{array}{l}6.3 \pm 0.3 \\
(0.57) \\
\end{array}$ \\
\hline \multirow{4}{*}{ 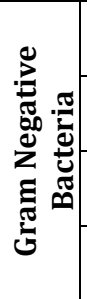 } & Escherichia coli & $\begin{array}{l}7.6 \pm 0.3 \\
(0.57)\end{array}$ & $\begin{array}{l}14.3 \pm 0.3 \\
(0.57)\end{array}$ & $\begin{array}{l}6.3 \pm 0.3 \\
(0.57)\end{array}$ & $\begin{array}{l}6.6 \pm 0.3 \\
(0.57)\end{array}$ & $\begin{array}{l}12.3 \pm 0.3 \\
(0.57)\end{array}$ & $\begin{array}{l}6.6 \pm 0.3 \\
(0.57)\end{array}$ \\
\hline & Klebsiella pneumonia & $\begin{array}{l}10.6 \pm 0.3 \\
(0.57)\end{array}$ & $\begin{array}{l}15.3 \pm 0.6 \\
(0.57)\end{array}$ & $\begin{array}{l}6.3 \pm 0.33 \\
(0.57)\end{array}$ & $\begin{array}{l}6.6 \pm 0.3 \\
(0.57)\end{array}$ & $\begin{array}{l}22.3 \pm 0.3 \\
(0.57)\end{array}$ & $\begin{array}{l}6.3 \pm 0.3 \\
(0.57) \\
\end{array}$ \\
\hline & $\begin{array}{l}\text { Pseudomonas } \\
\text { aeruginosa }\end{array}$ & $\begin{array}{l}7.6 \pm 0.3 \\
(0.57) \\
\end{array}$ & $\begin{array}{l}15.3 \pm 0.6 \\
(0.57)\end{array}$ & $\begin{array}{l}6.3 \pm 0.3 \\
(0.57) \\
\end{array}$ & $\begin{array}{l}6.6 \pm 0.3 \\
(0.57) \\
\end{array}$ & $\begin{array}{l}9.3 \pm 0.6 \\
(0.57) \\
\end{array}$ & $\begin{array}{l}6.3 \pm 0.3 \\
(0.57) \\
\end{array}$ \\
\hline & Proteus vulgaris & $\begin{array}{l}10.6 \pm 0.3 \\
(0.57)\end{array}$ & $\begin{array}{l}15.3 \pm 0.6 \\
(0.57)\end{array}$ & $\begin{array}{l}6.6 \pm 0.3 \\
(0.57)\end{array}$ & $\begin{array}{l}6.6 \pm 0.3 \\
(0.57)\end{array}$ & $\begin{array}{l}16.3 \pm 0.3 \\
(0.57)\end{array}$ & $\begin{array}{l}6.3 \pm 0.3 \\
(0.57)\end{array}$ \\
\hline
\end{tabular}

Legend: SE: Successive extract; NSE: Non-Successive extract 


\section{Conclusion}

It's concluded that the antibacterial activity of $M$. charantia is based on several factors like the solvent used for extraction, plant part, concentration, method of extraction, etc. However, the isolation of the principal compound and structural elucidation would have yet to be achieved.

\section{Acknowledgments}

The authors are grateful to all staff members of the Pharmacognosy laboratory, A.M.U, Aligarh who assisted in the experiment.

\section{Declaration of interest}

The authors have no conflicts of interest. The authors alone are responsible for the content and writing of the paper.

\section{References}

1. Abid Mahmood, Ghazala Kaukab Raja, Tariq Mahmood, Muhammad Gulfraz and Azra Khanum. 2012. Isolation and characterization of antimicrobial activity conferring component(s) from seeds of bitter gourd (Momordica charantia). Journal of Medicinal Plants Research 6(4), 566-573. https://doi.org/10.5897/JMPR10.613

2. Adegbola Rachael Adebola, Akinbile Yaya Akinwale, Awotoye Jane Ariyo. 2016. Mormodica charantia Linn. A Potential Antibiotic and Anti-Fungal Drug International Journal of Pharmaceutical Science Invention, 5 (2), 21-27.

3. Bauer A.W, Kirby W.M.M, Sherries J.C, Turck M. 1996.Antibiotic susceptibility testing by a standardized single disk method. Am J Clinical Pathology, 45(4), 493-496.

4. Bush K, Jacoby G.A and Medeiros A.A. 1995.A functional classification scheme for $\beta$-lactamases and its correlation with molecular structure. Antimicrob Agents Chemotherapy, 39, 12111233. http://dx.doi.org/10.1128/aac.39.6.1211

5. CLSI .2006.Performance standards for antimicrobial susceptibility testing, Fifteenth Informational Supplement, CLSI document, M100S16, vol 26-3; M7-A7, vol 26-2; M2-A9, vol 26-1. Wayne, PA, US.

6. Debolina Ghosh. 2014.Does Bitter Melon (Momordica charantia) have Antibacterial Property? Journal of Food Processing \& Technology, 5,7. https://doi.org/10.4172/2157-7110.1000345

7. G. Leelaprakash, J. Caroline Rose, Gowtham B.M, Pradeep Krishna Javvaji, Shivram Prasad. 2011.In vitro Antimicrobial and Antioxidant Activity of Momordica Charantia Leaves. Pharmacophore, 2 (4), 207215.

8. Gulsum Yaldiz, Nazim Sekeroglu, Muhittin Kulak and Gurkan Demirkol. 2015. Antimicrobial activity and agricultural properties of bitter melon (Momordica charantia L.) grown in northern parts of Turkey: a case study for adaptation, Natural Product Research. Formerly Natural Product Letters, 29(6), 543-545. https://doi.org/10.1080/14786419.2014.949706

9. K. D Mwambete. 2009.The in vitro antimicrobial activity of fruit and leaf crude extracts of Momordica charantia: A Tanzania medicinal plant. African Health Sciences, 9(1). PMCID: PMC2932517

10. Luria Leslie Founou, Raspail Carrel Founou, and Sabiha Yusuf Essack. (2016) Antibiotic Resistance in the Food Chain: A Developing Country-Perspective. Front Microbiol, 7, 1881. https://doi.org/10.3389/fmicb.2016.01881

11. M. Asan Ozusaglam and K. Karakoca. 2013. Antimicrobial and antioxidant activities of Momordica charantia from Turkey. African Journal of Biotechnology, 12 (13), 1548-1558. http://dx.doi.org/10.5897/AJB2012.2932

12. Organization WH. WHO traditional medicine strategy 2002- 2005. 2002.

13. P. Supraja and R. Usha. 2013. Antibacterial and Phytochemical Screening from Leaf and Fruit Extracts of Momordica charantia. Int J Pharm Bio Sci, 4(1), (B) 787 - 793.
14. Poole K. 2011. Pseudomonas aeruginosa: resistance to the max. Frontier Microbiology, 65, 1-13 http://dx.doi.org/10.3389/fmicb.2011.00065

15. S. B. Mada1, A. Garba1, H. A. Mohammed, A. Muhammad, A. Olagunju and A. B. Muhammad. 2013. Antimicrobial activity and phytochemical screening of aqueous and ethanol extracts of Momordica charantia L. leaves. Journal of Medicinal Plants Research, 7(10), 579-586. http://dx.doi.org/10.5897/JMPR012.1161

16. Sabahat Saeed and Perween Tariq. 2005. Antibacterial Activities of Mentha piperita, Pisum sativum and Momordica charantia. Pak. J. Bot., 37(4), 997-1001.

17. Sharma V.K, Johnson N, Cizmas L, Mc Donald T.J, Kim H. 2016. A review of the influence of treatment strategies on antibiotic resistant bacteria and antibiotic resistance genes. Chemosphere, 150,702714. http://dx.doi.org/10.1016/j.chemosphere.2015.12.084

18. Shuo Jia, Mingyue Shen, Fan Zhang and Jianhua Xie. 2017. Recent Advances in Momordica charantia: Functional Components and Biological Activities. Int. J. Mol. Sci, 18, 2555. http://dx.doi.org/10.3390/ijms18122555

19. Yang Lin Yeo, Yin Yin Chia, Chin Hong Lee, Heng Sheng Sow, Wai Sum Yap. 2014. Effectiveness of Maceration Periods with Different Extraction Solvents on in-vitro Antimicrobial Activity from Fruit of Momordica charantia L. Journal of Applied Pharmaceutical Science, 4 (10), 016-023. http://dx.doi.org/10.7324/JAPS.2014.40104

20. Yeh-Lin Lu, Yuh-Hwa Liu, Wen-Li Liang, Jong-Ho Chyuan, Kur-Ta Cheng, Hong-Jen Liang and Wen-Chi Hou. 2011.Antibacterial and cytotoxic activities of different wild bitter gourd cultivars (Momordica charantia L. var. abbreviata Seringe). Botanical Studies, 52,427-434.

21. YinYin Chia and WaiSum Yap.2011.In vitro Antimicrobial Activity of Hexane: Petroleum Ether Extracts from Fruits of Momordica charantia L. International Journal of Pharmaceutical \& Biological Archives, 2(3), 868-873. http://dx.doi.org/10.7324/JAPS.2014.40104 